\title{
A MESH TRANSFORMATION METHOD FOR COMPUTING MICROSTRUCTURES
}

\author{
ZHIPING LI \\ SCHOOL OF MATHEMATICAL SCIENCES, PEKING UNIVERSITY, \\ BEIJING 100871, P.R.CHINA
}

\begin{abstract}
A numerical method is established to solve the problem of minimizing a nonquasiconvex potential energy. Convergence of the method is proved both in the case on its own and in the case when it is combined with a weak boundary condition. Numerical examples are given to show that the method, especially when applied together with a continuation method and some other numerical techniques, is not only successful and efficient in solving problems with laminated microstructures but also capable of computing more complicated microstructures.
\end{abstract}

\section{INTRODUCTION}

Microstructure is a phenomenon found in many physical problems, such as those which involve phase transitions and hysteresis [1,2]. A related mathematical problem is to minimize a potential energy

$$
F(u ; \Omega)=\int_{\Omega} f(x, u(x), \nabla u(x)) d x
$$

with nonquasiconvex energy density $f: \Omega \times R^{m} \times R^{m n} \rightarrow R^{1}$ on a set of admissible functions

$$
\mathbb{U}\left(u_{0} ; \Omega\right)=\left\{u \in W^{1, p}\left(\Omega ; R^{m}\right): u=u_{0}, \text { on } \partial \Omega\right\},
$$

where $\Omega \subset R^{n}$ is a bounded open set with a Lipschitz continuous boundary $\partial \Omega$ and $1<p<\infty$.

It is well known that such a variational problem fails, in general, to have a solution, and the minimizing sequences of the potential energy can develop finer and finer oscillations and lead to microstructures $[1,3,4]$, which are characterized by the Young measures [5]. To compute the microstructures, or rather the highly

1991 Mathematics Subject Classification. 65K10,65N12,65N30,49J45,49M20.

Key words and phrases. nonquasiconvex, microstructure, minimizing sequence, mesh transformation, finite element solutions, convergence.

The research was supported in part by the Special Funds for Major State Basic Research Projects (G1999032802), NSFC and RFDP of China. 
oscillating minimizing sequences of $F(\cdot, \Omega)$ in $\mathbb{U}\left(u_{0} ; \Omega\right)$, is challenging and is of great interests both in theory and in applications. Great progress has been made in recent years, and many numerical methods have been established (see [6]-[24] among many others, see [15] for more references).

It is known that, by applying an iterative method with a piecewise affine finite element approximation, the numerical computation of microstructures for a problem involving an inhomogeneous energy density and nonlinear boundary conditions can be transformed into the numerical computations of microstructures for a group of related homogeneous problems, i.e. the problems with energy densities of the form $f(\nabla u)$ and with linear boundary conditions. Hence, It is of essential importance to establish efficient numerical methods for computing microstructures for homogeneous problems, which is the focus of this paper.

For problems involving microstructures, numerical analyses and experiments revealed that the numerical results often depend strongly on the mesh and shape functions, and can sometimes lead to pseudo-microstructures [4, 10, 15]. A rotational transformation method established by Li [21] somehow reduces the mesh dependence of the numerical results, and it turns out to be successful in computing laminated microstructures. In the present paper, the idea of the rotational transformation method is further extended into a mesh transformation method where the rotational transformation of the mesh is replaced by a piecewise linear transformation. Basically the mesh transformation method gives more freedom in searching for a minimizer of the corresponding discrete problem and allows the mesh to be aligned with the interfaces between phases or phase variants, for example the mesh can be transformed to fit arbitrary volume fractions of a simple laminate (see section 3), and thus not only reduces further the mesh dependence but also reduces the possibility of being trapped into a local minimizer. Furthermore, compared with the rotational transformation method, the mesh transformation method provides more flexibility for the computation of microstructures which are not simple laminates (see section 3 and see [24] for more involved applications).

In section 2, the mesh transformation method is established and analyzed, convergence of the method is proved. We also consider to replace the "hard" boundary condition $\left.u\right|_{\partial D}=A x$ by adding a boundary integral term $\beta \int_{\partial D} \mid u-$ $A x \mid d x$ to the potential energy. The mesh transformation method combined with this technique shows better performance in the numerical experiments and is also proved to be convergent. The mesh transformation method combines with more subtle boundary technique for periodic boundary conditions can be found 
in [23]. In section 3 , the implementation of the mesh transformation method and the applications of some other techniques, such as the incremental crystallization method [21] and the continuation method, are discussed, and the numerical results for two model problems are given.

\section{ThE METHOD AND ITS ANALYSIS}

Let $\Omega \subset R^{n}$ be a bounded open set with a Lipschitz continuous boundary. Let $f: R^{m n} \rightarrow R^{1}$ be a continuous function which satisfies the following hypotheses for a constant $p>1$ :

$$
\begin{aligned}
& \text { (h1): } \max \left\{0, a_{1}+b_{1}|\xi|^{p}\right\} \leq f(\xi) \leq a_{2}+b_{2}|\xi|^{p}, \\
& \text { (h2): }|f(\xi)-f(\eta)| \leq C\left(1+|\xi|^{p-1}+|\eta|^{p-1}\right)|\xi-\eta|,
\end{aligned}
$$

where $a_{1} \in R^{1}, a_{2}>0, b_{2} \geq b_{1}>0$ and $C>0$ are constants. Consider the problem of minimizing the functional

$$
F(u ; \Omega)=\int_{\Omega} f(\nabla u(x)) d x
$$

on a set of admissible functions with a linear boundary condition

$$
\mathbb{U}(A ; \Omega)=\left\{u \in W^{1, p}\left(\Omega ; R^{m}\right): u(x)=A x, \text { on } \partial \Omega\right\},
$$

where $A \in R^{m n}$ is a given matrix, which may be assumed to be 0 if $f(\cdot)$ is replaced by $f(A+\cdot)(c . f .[8])$, however to help the readers to see clearly how the matrix $A$ is involved in the computation we simply leave it as it is.

Without loss of generality, assume

$$
\Omega \subset B(0 ; 1),
$$

where $B(0 ; 1)=\left\{x \in R^{n}:\|x\|_{2}<1\right\}$ is the open unit ball of $R^{n}$. Define

$$
D=(-1,1)^{n} .
$$

We have obviously

$$
\Omega \subset B(0 ; 1) \subset B(0 ; \sqrt{n})=\bigcap_{R \in S O(n)} R(D),
$$

where $S O(n)$ is the set of all $n \times n$ rotational transformation matrices $R$ with the determinant $\operatorname{det} R=1$. The hypercube $D$ will serve as the working domain for our numerical computation. 
For a given $R \in S O(n)$, define

$T(D ; R)=\left\{\right.$ bijections $L: D \rightarrow R(D) \mid L \in W^{1, \infty}(D), L^{-1} \in W^{1, \infty}(R(D))$ and $\operatorname{det} \nabla L>0$, a.e. in $D\}$,

and

$$
T(D)=\{L \in T(D ; R) \text { : for some } R \in S O(n)\} .
$$

Lemma 2.1. For any $L \in T(D)$, we have

$$
\inf _{u \in \mathbb{U}(A ; L(D))} F(u ; L(D))=\inf _{u \in \mathbb{U}(A ; \Omega)} \frac{1}{\operatorname{meas}(\Omega)} F(u ; \Omega),
$$

where meas $(\cdot)$ is the Lebesgue measure in $R^{n}$.

Proof. It is well known (cf. $[25,26])$ that

$$
Q f(A)=\inf _{u \in \mathbb{U}\left(A ; \Omega^{\prime}\right)} \frac{1}{\operatorname{meas}\left(\Omega^{\prime}\right)} F\left(u ; \Omega^{\prime}\right)
$$

for all bounded open set $\Omega^{\prime} \subset R^{n}$, where $Q f(\cdot)$ is the quasiconvex envelope of $f(\cdot)[25,27]$. Thus the lemma follows, since meas $(D)=\operatorname{meas}(L(D))=1$ for all $L \in T(D)$.

Lemma 2.2. For any $L \in T(D)$ and $\bar{u} \in \mathbb{U}(A ; L(D))$, let $u(x): D \rightarrow R^{m}$ be defined by

$$
u(x)=\bar{u}(L(x))-A L(x) .
$$

Then $u \in \mathbb{U}(0 ; D)$ and

$$
\int_{D} f\left(A+\nabla u(x)(\nabla L(x))^{-1}\right) \operatorname{det} \nabla L(x) d x=F(\bar{u} ; L(D)) .
$$

Proof. The relation $u \in \mathbb{U}(0 ; D)$ follows directly from (2.7) and (2.2). By a change of variables, we have (2.8).

As a direct corollary of lemmas 2.1 and 2.2, we have

Corollary 2.1. Let $L \in T(D)$. Define

$$
\left.F(u, L ; D)=\int_{D} f\left(A+\nabla u(x)(\nabla L(x))^{-1}\right)\right) \operatorname{det} \nabla L(x) d x .
$$

Then

$$
\inf _{u \in \mathbb{U}(0 ; D)} F(u, L ; D)=\inf _{u \in \mathbb{U}(A ; \Omega)} \frac{1}{\operatorname{meas}(\Omega)} F(u ; \Omega) .
$$


Let $\mathfrak{T}_{h}$ be regular triangulations of $D$ with mesh sizes $h$ [28]. Let

$$
\begin{aligned}
T_{h}(D) & =\left\{L \in T(D):\left.L\right|_{K} \text { is affine for all } K \in \mathfrak{T}_{h}\right\}, \\
\mathbb{U}_{h} & =\left\{u \in(C(\bar{D}))^{m}:\left.u\right|_{K} \text { is affine } \forall K \in \mathfrak{T}_{h}\right\}
\end{aligned}
$$

and

$$
\mathbb{U}_{h}(A ; D)=\left\{u \in \mathbb{U}_{h}:\left.u\right|_{\partial D}=A x\right\} .
$$

In the mesh transformation method, we solve the following discrete problem :

$$
(D P) \quad\left\{\begin{array}{l}
\text { find }(u, L) \in \mathbb{U}_{h}(0 ; D) \times T_{h}(D) \text { such that } \\
F(u, L ; D)=\inf _{\left(u^{\prime}, L^{\prime}\right) \in \mathbb{U}_{h}(0 ; D) \times T_{h}(D)} F\left(u^{\prime}, L^{\prime} ; D\right) .
\end{array}\right.
$$

Theorem 2.1. Let $\lim _{i \rightarrow \infty} h_{i}=0$ and $\lim _{i \rightarrow \infty} \varepsilon_{i}=0$. Let the functions $\left(u_{h_{i}}, L_{h_{i}}\right)$ $\in \mathbb{U}_{h_{i}}(0 ; D) \times T_{h_{i}}(D)$ be a sequence of approximate solutions to (DP) (see remark 2.1) with

$$
F\left(\bar{u}_{h_{i}}, L_{h_{i}}(D)\right) \leq \inf _{(u, L) \in \mathbb{U}_{h_{i}}(0 ; D) \times T_{h_{i}}(D)} F(u, L ; D)+\varepsilon_{i} .
$$

Then

$$
\lim _{i \rightarrow \infty} F\left(\bar{u}_{h_{i}}, L_{h_{i}}(D)\right)=\lim _{i \rightarrow \infty} F\left(u_{h_{i}}, L_{h_{i}} ; D\right)=\inf _{u \in \mathbb{U}(A ; D)} F(u, D),
$$

where $\bar{u}_{h_{i}}(x)=u_{h_{i}}\left(L_{h_{i}}^{-1}(x)\right)+A L_{h_{i}}^{-1}(x)($ see $(2.7))$.

Proof. It follows from lemma 2.1 that

$$
\inf _{u \in \mathbb{U}(A ; D)} F(u ; D)=\inf _{u \in \mathbb{U}(0 ; D)} F\left(u, L_{h_{i}} ; D\right) \leq F\left(u_{h_{i}}, L_{h_{i}} ; D\right) .
$$

On the other hand, for the identity $I: D \rightarrow D$, we have

$$
F\left(u_{h_{i}}, L_{h_{i}} ; D\right) \leq \inf _{u \in \mathbb{U}_{h_{i}}(0 ; D)} F(u, I ; D)+\varepsilon_{i} .
$$

By the standard finite element approximation theory [28], we have

$$
\lim _{h_{i} \rightarrow 0} \inf _{u \in \mathbb{U}_{h_{i}}(0 ; D)} F(u, I ; D)=\inf _{u \in \mathbb{U}(0 ; D)} F(u, I ; D) .
$$

Combining (2.18) with (2.16), (2.17), (2.8) and (2.10), we obtain (2.15).

Corollary 2.2. As a consequence of lemma 2.1, lemma 2.2 and theorem 2.1, we have

$$
\begin{aligned}
Q f(A) & =\frac{1}{\operatorname{meas}(\Omega)} \inf _{u \in \mathbb{U}(A ; \Omega)} F(u ; \Omega) \\
& =\lim _{h \rightarrow 0} \inf _{(u, L) \in \mathbb{U}_{h}(0 ; D) \times T_{h}(D)} F(u, L ; D),
\end{aligned}
$$

where $Q f(\cdot)$ is the quasiconvex envelope of $f(\cdot)[25,26,27]$. 
Let $\left(u_{h}, L_{h}\right) \in \mathbb{U}_{h}(0 ; D) \times T_{h}(D)$ be a sequence of solutions to (DP) with $h \rightarrow 0$, then they can be used to construct a minimizing sequence of $F(\cdot, \Omega)$ in $\mathbb{U}(A ; \Omega)$ in the following standard way (see for example [26]). Assume $h \ll 1$, let $R_{h} \in S O(n)$ be such that $L_{h} \in T\left(D ; R_{h}\right)$. Define

$$
\Omega(h)=\left\{x: x \in h\left(2 R_{h} z+L_{h}(D)\right) \subset \Omega \text { for some } z \in \mathbb{Z}^{n}\right\},
$$

where $\mathbb{Z}$ is the set of all integers. Let $\varphi_{h}: \Omega \rightarrow R^{1}$ be a truncation function defined by

$$
\varphi_{h}(x)= \begin{cases}1, & \text { if } x \in \Omega_{h} \\ 0, & \text { otherwise }\end{cases}
$$

Define $\hat{u}_{h}: \Omega \rightarrow R^{m}$ by

$$
\hat{u}_{h}(x)=\varphi_{h}(x) h u_{h}\left(L_{h}^{-1}\left(h^{-1} x\right)\right)+A x,
$$

where $L_{h}^{-1}$ is defined on the whole space of $R^{n}$ by a periodic extension

$$
L_{h}^{-1}\left(x^{\prime}\right)=L_{h}^{-1}(x), \forall x^{\prime} \in R^{n}, x \in L_{h}(\bar{D}) \text { and } \frac{1}{2}\left(x^{\prime}-x\right) \in R_{h}\left(\mathbb{Z}^{n}\right) .
$$

Theorem 2.2. Let $\left(u_{h}, L_{h}\right) \in \mathbb{U}_{h}(0 ; D) \times T_{h}(D)$ be a sequence of approximate solutions to (DP) (see theorem 2.1) with $h \rightarrow 0$. Let $\hat{u}_{h}$ be defined by (2.19). Then

$$
\hat{u}_{h} \in \mathbb{U}(A ; \Omega), \quad \hat{u}_{h} \rightarrow A x \text { in } L^{p}\left(\Omega ; R^{m}\right)
$$

and

$$
\lim _{h \rightarrow 0} F\left(\hat{u}_{h} ; \Omega\right)=\inf _{u \in \mathbb{U}(A ; \Omega)} F(u ; \Omega) .
$$

Proof. The relation (2.21) follows directly from the definition of $\hat{u}_{h}$. Let $R_{h} \in$ $S O(n)$ be such that $L_{h} \in T\left(D ; R_{h}\right)$, and let $\mathbb{Z}_{h}=\left\{z \in \mathbb{Z}^{n}: h\left(2 R_{h} z+L_{h}(D)\right) \subset\right.$ $\Omega\}$. Then, a straight forward calculation yields

$$
\begin{aligned}
F(\hat{u} ; \Omega) & =F(A x ; \Omega \backslash \Omega(h))+F\left(\hat{u}_{h} ; \Omega(h)\right) \\
& =F(A x ; \Omega \backslash \Omega(h))+\sum_{z \in \mathbb{Z}_{h}} F\left(\hat{u}_{h} ; h L_{h}(D)\right) \\
& =F(A x ; \Omega \backslash \Omega(h))+\sum_{z \in \mathbb{Z}_{h}} h^{n} F\left(u_{h}, L_{h} ; D\right) .
\end{aligned}
$$

Since $\lim _{h \rightarrow 0}$ meas $(\Omega \backslash \Omega(h))=0$ and

$$
\sum_{z \in \mathbb{Z}_{h}} h^{n}=\operatorname{meas}(\Omega(h))
$$

(2.22) follows from (2.23) and corollary 2.2. 
As a consequence of theorem 2.2, by solving the discrete problems (DP) (see (2.14)), we obtain a minimizing sequence $\left\{\hat{u}_{h}\right\}$ (see $(2.19)$ ) of $F(\cdot ; \Omega)$ in $\mathbb{U}(A ; \Omega)$. However, in numerical computations, the strict satisfaction of the boundary condition $\left.u\right|_{\partial D}=0$ causes energy accumulation in the area near the boundary and can even sometimes cause difficulties in the formation of oscillations, or in other words numerical microstructures. To reduce such a boundary effect, we consider, instead of the problem (DP), the following discrete problem :

$$
(D P I) \quad\left\{\begin{array}{l}
\text { find }(u, L) \in \mathbb{U}_{h}(D) \times T_{h}(D) \text { such that } \\
F_{\alpha, r}(u, L ; D)=\inf _{\left(u^{\prime}, L^{\prime}\right) \in \mathbb{U}_{h}(D) \times T_{h}(D)} F_{\alpha, r}\left(u^{\prime}, L^{\prime} ; D\right),
\end{array}\right.
$$

where $\alpha>0$ and $0<r<p$ are parameters to be given in the computation and

$$
F_{\alpha, r}(u, L ; D)=F(u, L ; D)+\alpha h^{-r} \int_{\partial L(D)}\left|u\left(L^{-1}(x)\right)\right|^{p} d x .
$$

It is obvious that for all $\alpha>0$ and $0<r<p$

$$
\inf _{\left(u^{\prime}, L^{\prime}\right) \in \mathbb{U}_{h}(D) \times T_{h}(D)} F_{\alpha, r}\left(u^{\prime}, L^{\prime} ; D\right) \leq \inf _{\left(u^{\prime}, L^{\prime}\right) \in \mathbb{U}_{h}(0 ; D) \times T_{h}(D)} F\left(u^{\prime}, L^{\prime} ; D\right) .
$$

Lemma 2.3. Let $\left(\tilde{u}_{h_{i}}, L_{h_{i}}\right) \in \mathbb{U}_{h_{i}}(D) \times T_{h_{i}}(D)$ be a sequence of solutions (see remark 2.1) to (DPI) with $\lim _{i \rightarrow \infty} h_{i}=0$. Then,

$$
\lim _{i \rightarrow \infty} F\left(\tilde{u}_{h_{i}}, L_{h_{i}} ; D\right)=\lim _{i \rightarrow \infty} F_{\alpha, r}\left(\tilde{u}_{h_{i}}, L_{h_{i}} ; D\right)=\inf _{u \in \mathbb{U}(A ; D)} F(u ; D),
$$

and the sequence $\left\{\left|\nabla \tilde{u}_{h_{i}}\left(\nabla L_{h_{i}}\right)^{-1}\right|^{p} \operatorname{det} \nabla L_{h_{i}}\right\}_{i=1}^{\infty}$ are equi-uniformly integral continuous [29] in the sense that for any given $\varepsilon>0$, there exists $\delta(\varepsilon)>0$ such that for all $i$ and any measurable set $E \subset D$

$$
\int_{E}\left|\nabla \tilde{u}_{h_{i}}(x)\left(\nabla L_{h_{i}}(x)\right)^{-1}\right|^{p} \operatorname{det} \nabla L_{h_{i}}(x) d x<\varepsilon, \text { if meas }\left(L_{h_{i}}(E)\right)<\delta .
$$

Proof. Let $R_{h_{i}} \in S O(n)$ be such that $L_{h_{i}} \in T\left(D ; R_{h_{i}}\right)$. Since $S O(n)$ is a compact set in $R^{2 \times 2}$, without loss of generality, we may assume that for some $R \in S O(n)$

$$
R_{h_{i}} \rightarrow R \quad \text { in } R^{2 \times 2}
$$

Denote $P_{h_{i}}=R R_{h_{i}}^{-1}$ and define $\bar{u}_{h_{i}}: R(D) \rightarrow R^{m}$ by

$$
\bar{u}_{h_{i}}(x)=A x+\tilde{u}_{h_{i}}\left(L_{h_{i}}^{-1}\left(P_{h_{i}}^{-1} x\right)\right) .
$$


Then, by a change of variables of integration and noticing that $\operatorname{det} P_{h_{i}}=1$, we have

$$
F\left(\bar{u}_{h_{i}} ; R(D)\right)=\int_{D} f\left(A+\nabla \tilde{u}_{h_{i}}(x)\left(\nabla L_{h_{i}}(x)\right)^{-1} P_{h_{i}}^{-1}\right) \operatorname{det} \nabla L_{h_{i}}(x) d x .
$$

It follows from (2.30) and (h1) that

$$
\begin{aligned}
\left|F\left(\bar{u}_{h_{i}} ; R(D)\right)\right| & \leq C \int_{D}\left(1+\left|\nabla \tilde{u}_{h_{i}}(x)\left(\nabla L_{h_{i}}(x)\right)^{-1}\right|^{p}\right) \operatorname{det} \nabla L_{h_{i}}(x) d x \\
& \leq C\left(1+F\left(\tilde{u}_{h_{i}}, L_{h_{i}} ; D\right)\right) .
\end{aligned}
$$

By (h1), (h2), (2.26),(2.31) and corollary 2.2, we have

$$
\begin{aligned}
& \left|F\left(\bar{u}_{h_{i}} ; R(D)\right)-F\left(\tilde{u}_{h_{i}}, L_{h_{i}} ; D\right)\right| \\
\leq & C \int_{D}\left(1+\left|A+\nabla \tilde{u}_{h_{i}}(x)\left(\nabla L_{h_{i}}(x)\right)^{-1} P_{h_{i}}^{-1}\right|^{p}\right. \\
& \left.+\left|A+\nabla \tilde{u}_{h_{i}}(x)\left(\nabla L_{h_{i}}(x)\right)^{-1}\right|^{p}\right)\left|P_{h_{i}}-I\right| \operatorname{det} \nabla L_{h_{i}}(x) d x \\
\leq & C\left|P_{h_{i}}-I\right|\left(1+F\left(\bar{u}_{h_{i}} ; R(D)\right)+F\left(\tilde{u}_{h_{i}}, L_{h_{i}} ; D\right)\right) \leq C\left|P_{h_{i}}-I\right| .
\end{aligned}
$$

(2.26), (2.28), (2.32) and corollary 2.1-2.2 imply

$$
\limsup _{i \rightarrow \infty} F\left(\bar{u}_{h_{i}} ; R(D)\right) \leq \inf _{u \in \mathbb{U}(A ; R(D))} F(u ; R(D)) .
$$

It follows from (2.33) and (h1) that $\left\{\bar{u}_{h_{i}}\right\}_{i=1}^{\infty}$ are bounded in $W^{1, p}\left(R(D) ; R^{m}\right)$. Thus, without loss of generality, we may assume [25] that

$$
\bar{u}_{h_{i}} \rightarrow u_{\infty}, \quad \text { in } W^{1, p}\left(R(D) ; R^{m}\right),
$$

Where ' - ' means 'converges weakly to'. Since, by (2.25), (2.26) and (2.29),

$$
\int_{\partial D}\left|\bar{u}_{h_{i}}-A x\right|^{p} d x=\int_{\partial L_{h_{i}}(D)}\left|\tilde{u}_{h_{i}}\left(L_{h_{i}}^{-1}(x)\right)\right|^{p} d x \leq C h_{i}^{r},
$$

(2.34) and the Sobolev imbedding theorem [30] imply that $u_{\infty} \in \mathbb{U}(A ; R(D))$. Let

$$
Q F(u ; R(D))=\int_{R(D)} Q f(\nabla u(x)) d x, \text { for } u \in W^{1, p}\left(R(D) ; R^{m}\right) .
$$

Then, by (2.34), we have $[26,31]$

$$
Q F\left(u_{\infty} ; R(D)\right) \leq \liminf _{i \rightarrow \infty} Q F\left(\bar{u}_{h_{i}} ; R(D)\right) \leq \liminf _{i \rightarrow \infty} F\left(\bar{u}_{h_{i}} ; R(D)\right) .
$$

By (2.33), (2.36) and

$$
\inf _{u \in \mathbb{U}(A ; R(D))} Q F(u ; R(D))=\inf _{u \in \mathbb{U}(A ; R(D))} F(u ; R(D))
$$


(cf. $[26,31]$ ), we have

$$
\inf _{u \in \mathbb{U}(A ; R(D))} Q F(u ; R(D))=Q F\left(u_{\infty} ; R(D)\right)=\lim _{i \rightarrow \infty} Q F\left(\bar{u}_{h_{i}} ; R(D)\right) .
$$

Now, (2.27) follows from (2.26), (2.28), (2.32), (2.33), (2.37) and (2.38). It is a result given by Kinderlehrer and Pedregal $[32,33]$ that $(2.34),(2.38)$ and (h1) imply that $\left\{\left|\nabla \bar{u}_{h_{i}}\right|^{p}\right\}_{i=1}^{\infty}$ are equi-uniformly integral continuous, and thus the lemma holds as a consequence.

Lemma 2.4. (Poincaré-Friedrichs inequality) For $d<1$ and $R \in S O(n)$, let $D(d, R)=\{x \in R(D): \operatorname{dist}(x, \partial R(D))<d\}$. Let $u \in W^{1, p}\left(R(D) ; R^{m}\right)$. Then, we have

$$
\|u\|_{0, p, D(d, R)}^{p} \leq 2^{p-1}\left(\|u\|_{0, p, \partial R(D)}^{p}+\frac{1}{p} d^{p}|u|_{1, p, D(d, R)}^{p}\right) .
$$

Proof. Without loss of generality, we assume that $R=I$. Let $x^{\prime} \in[-1,1]^{n-1}$. For $x_{n} \in(-1,-1+d)$ and $u \in C^{1}\left(\bar{D} ; R^{m}\right)$, we have

$$
\begin{aligned}
\left|u\left(x^{\prime}, x_{n}\right)\right|^{p} & =\left|u\left(x^{\prime},-1\right)+\int_{-1}^{x_{n}} \frac{\partial u\left(x^{\prime}, x_{n}\right)}{\partial x_{n}} d x_{n}\right|^{p} \\
& \leq 2^{p-1}\left(\left|u\left(x^{\prime},-1\right)\right|^{p}+\left|\int_{-1}^{x_{n}} \frac{\partial u\left(x^{\prime}, x_{n}\right)}{\partial x_{n}} d x_{n}\right|^{p}\right) \\
& \leq 2^{p-1}\left(\left|u\left(x^{\prime},-1\right)\right|^{p}+\left(x_{n}+1\right)^{p-1} \int_{-1}^{x_{n}}\left|\frac{\partial u\left(x^{\prime}, x_{n}\right)}{\partial x_{n}}\right|^{p} d x_{n}\right) .
\end{aligned}
$$

Integrating over $D^{-}(d, n)=\left\{x \in D(d): x_{n} \in(-1,-1+d)\right\}$, we obtain

$$
\|u\|_{0, p, D^{-}(d, n)}^{p} \leq 2^{p}\left(\|u\|_{0, p, \partial D_{n}^{-}}^{p}+\frac{1}{p} d^{p}|u|_{1, p, D^{-}(d, n)}^{p}\right),
$$

where $\partial D_{n}^{-}=\left\{x \in \partial D: x_{n}=-1\right\}$. Applying the above argument on the other parts of $\partial D$, summing up the obtained results and considering that $C^{1}\left(\bar{D} ; R^{m}\right)$ is dense in $W^{1, p}\left(D ; R^{m}\right)$ [30], we have the conclusion.

Theorem 2.3. Let $\left(\tilde{u}_{h_{i}}, L_{h_{i}}\right) \in \mathbb{U}_{h_{i}} \times T_{h_{i}}(D)$ be a sequence of solutions (see remark 2.1) to (DPI) with $\lim _{i \rightarrow \infty} h_{i}=0$. Let $u_{h_{i}}: D \rightarrow R^{m}$ be defined by

$$
u_{h_{i}}(x)=\psi_{h_{i}}\left(L_{h_{i}}(x)\right) \tilde{u}_{h_{i}}(x),
$$

where $\psi_{h_{i}} \in C^{\infty}\left(L_{h_{i}}(\bar{D})\right)$ are truncation functions satisfying

$$
\psi_{h_{i}}(x)= \begin{cases}0, & \text { if } x \in \partial L_{h_{i}}(D), \\ 1, & \text { if } \operatorname{dist}\left(x, \partial L_{h_{i}}(D)\right) \geq h_{i}^{s},\end{cases}
$$




$$
\left|\nabla \psi_{h_{i}}(x)\right| \leq C h_{i}^{-s}, \quad \forall x \in L_{h_{i}}(\bar{D}),
$$

and where $C>1$ and $0<s<r / p$ are constants. Then $u_{h_{i}} \in \mathbb{U}(0 ; D)$ and

$$
\lim _{i \rightarrow \infty} F\left(u_{h_{i}}, L_{h_{i}} ; D\right)=\inf _{u \in \mathbb{U}(A ; D)} F(u ; D)
$$

Proof. It is obvious that $u_{h_{i}} \in \mathbb{U}(0 ; D)$. Let

$$
L_{h_{i}}^{s}(D)=\left\{x \in L_{h_{i}}(D): \operatorname{dist}\left(x, \partial L_{h_{i}}(D)\right) \geq h_{i}^{s}\right\}
$$

and denote $L_{h_{i}}^{-1}\left(L_{h_{i}}(D) \backslash L_{h_{i}}^{s}(D)\right)=D \backslash L_{h_{i}}^{-1}\left(L_{h_{i}}^{s}(D)\right)$ by $D_{h_{i}}^{s}$. Since $u_{h_{i}}=\tilde{u}_{h_{i}}$ on $D \backslash D_{h_{i}}^{s}$, it follows from (h2) and the Hölder's inequality [30] that

$$
\begin{aligned}
& \left|F\left(u_{h_{i}}, L_{h_{i}} ; D\right)-F\left(\tilde{u}_{h_{i}}, L_{h_{i}} ; D\right)\right|=\left|F\left(u_{h_{i}}, L_{h_{i}} ; D_{h_{i}}^{s}\right)-F\left(\tilde{u}_{h_{i}}, L_{h_{i}} ; D_{h_{i}}^{s}\right)\right| \\
\leq & C\left[\left(\operatorname{meas}\left(L_{h_{i}}\left(D_{h_{i}}^{s}\right)\right)\right)^{\frac{p-1}{p}}+\left(\int_{D_{h_{i}}^{s}}\left|A+\nabla u_{h_{i}}\left(\nabla L_{h_{i}}\right)^{-1}\right|^{p} \operatorname{det} \nabla L_{h_{i}} d x\right)^{\frac{p-1}{p}}\right. \\
& \left.+\left(\int_{D_{h_{i}}^{s}}\left|A+\nabla \tilde{u}_{h_{i}}\left(\nabla L_{h_{i}}\right)^{-1}\right|^{p} \operatorname{det} \nabla L_{h_{i}} d x\right)^{\frac{p-1}{p}}\right] \\
& \cdot\left(\int_{D_{h_{i}}^{s}}\left|\left(\nabla u_{h_{i}}-\nabla \tilde{u}_{h_{i}}\right)\left(\nabla L_{h_{i}}\right)^{-1}\right|^{p} \operatorname{det} \nabla L_{h_{i}} d x\right)^{\frac{1}{p}} \\
= & C\left(I_{1}^{\frac{p-1}{p}}+I_{2}^{\frac{p-1}{p}}+I_{3}^{\frac{1}{p}}\right) I_{4}^{\frac{1}{p}} .
\end{aligned}
$$

It follows from (2.41), (2.42) and the Hölder's inequality that

$$
\begin{aligned}
I_{4} & \leq C\left(\int_{D_{h_{i}}^{s}}\left|\nabla \tilde{u}_{h_{i}}\left(\nabla L_{h_{i}}\right)^{-1}\right|^{p} \operatorname{det} \nabla L_{h_{i}} d x+h_{i}^{-p s} \int_{L_{h_{i}}\left(D_{h_{i}}^{s}\right)}\left|\tilde{u}_{h_{i}}\left(L_{h_{i}}^{-1}(x)\right)\right|^{p} d x\right) \\
& \leq C\left(I_{21}+h_{i}^{-p s} I_{22}\right) \\
I_{2} & \leq C\left(I_{1}+I_{21}+h_{i}^{-p s} I_{22}\right)
\end{aligned}
$$

Since

$$
\operatorname{meas}\left(L_{h_{i}}\left(D_{h_{i}}^{s}\right)\right) \leq C h_{i}^{s}
$$

it follows from lemma 2.3 that

$$
\lim _{i \rightarrow \infty} I_{1}=\lim _{i \rightarrow \infty} I_{21}=\lim _{i \rightarrow \infty} I_{3}=0 .
$$

By lemma 2.4,

$$
I_{22} \leq C\left(\int_{\partial L_{h_{i}}(D)}\left|\tilde{u}_{h_{i}}\left(L_{h_{i}}^{-1}(x)\right)\right|^{p} d x+h_{i}^{p s} \int_{D_{h_{i}}^{s}}\left|\nabla \tilde{u}_{h_{i}}\left(\nabla L_{h_{i}}\right)^{-1}\right|^{p} \operatorname{det} \nabla L_{h_{i}} d x\right) .
$$


By (2.25), (2.26) (2.47) and lemma 2.3, this gives

$$
\lim _{i \rightarrow \infty} h_{i}^{-p s} I_{22} \leq \lim _{i \rightarrow \infty} C\left(h_{i}^{r-p s}+\int_{D_{h_{i}}^{s}}\left|\nabla \tilde{u}_{h_{i}}\left(\nabla L_{h_{i}}\right)^{-1}\right|^{p} \operatorname{det} \nabla L_{h_{i}} d x\right)=0 .
$$

Thus, (2.43) follows as a consequence of (2.44)-(2.49) and lemm 2.3.

Theorem 2.4. Let $\left(\tilde{u}_{h}, L_{h}\right) \in \mathbb{U}_{h}(D) \times T_{h}(D)$ be a sequence of solutions (see remark 2.1) to (DPI) with $h \rightarrow 0$. Let $u_{h}: R^{n} \rightarrow R^{m}$ be defined by (2.40). Let $\hat{u}_{h}: \Omega \rightarrow R^{m}$ be defined by (2.19). Then

$$
\hat{u}_{h} \in \mathbb{U}(A ; \Omega), \text { and } \hat{u}_{h} \rightarrow A x \text { in } L^{p}\left(\Omega ; R^{m}\right)
$$

and

$$
\lim _{h \rightarrow 0} F\left(\hat{u}_{h} ; \Omega\right)=\inf _{u \in \mathbb{U}(A ; \Omega)} F(u ; \Omega) .
$$

Proof. With the same arguments as in the proof of theorem 2.2, and by theorem 2.3, we have the result.

Remark 2.1. The minima of (DP) and (DPI) may not be attainable. However, this is not of practical concern, since the results of this section remain valid as long as the numerical solutions to (DP) and (DPI) satisfy

$$
\lim _{h \rightarrow 0}\left(F\left(u_{h}, L_{h} ; \Omega\right)-\inf _{\left(u^{\prime}, L^{\prime}\right) \in \mathbb{U}_{h}(0 ; D) \times T_{h}(D)} F\left(u^{\prime}, L^{\prime} ; D\right)\right)=0
$$

and

$$
\lim _{h \rightarrow 0}\left(F_{\alpha, r}\left(\tilde{u}_{h}, L_{h} ; \Omega\right)-\inf _{\left(u^{\prime}, L^{\prime}\right) \in \mathbb{U}_{h}(D) \times T_{h}(D)} F_{\alpha, r}\left(u^{\prime}, L^{\prime} ; D\right)\right)=0
$$

respectively.

\section{Numerical Examples}

In applying the mesh transformation method established in section 2, We need to solve (DP) or (DPI) numerically. Since the numerical solutions are expected to converge weakly in $W^{1, p}\left(\Omega ; R^{m}\right)$ to the affine function $A x[26,21]$, to enhance the formation of such oscillations, we add a penalty term

$$
\beta h^{-q} \int_{D}\left|u_{h}(x)\right|^{p} \operatorname{det} \nabla L_{h}(x) d x
$$

to the potential energy (see also [21]) where $\beta>0$ and $0<q<p$ are parameters. An optimization method, for example gradient iterative methods $[6,10]$ and Methods using simulated annealing and Monte Carlo techniques [11, 12], can 
then be applied to solve the obtained discrete problems. In the following numerical examples, the conjugate gradient method is used. To increase the accuracy of the numerical approximation, after an initial convergent criterion is satisfied, the parameters $\alpha$ in (2.24) and $\beta$ in (3.1) can be reduced gradually as long as the integrals $\int_{\partial L_{h}(D)}\left|u_{h}(x)\right|^{p} d x$ and $\int_{D}\left|u_{h}(x)\right|^{p} \operatorname{det} \nabla L_{h}(x) d x$ do not increase too much (for example less than 3 times) [21] which is usually the case when a "good" oscillation pattern is formed. The incremental crystallization method [21] can also be applied in the optimization procedure, roughly speaking the optimization is restricted to a subsequently increasing subsets $D_{1} \subset D_{2} \subset \cdots \subset D_{k}=D$ of $D$ and the algorithm goes on to the next domain only after certain convergent criterion is satisfied. Another technique used in the following examples is the continuation method. The idea is that problems with topologically similar microstructures may be linked by a parameter and by tracing the varying microstructure we may very well obtain a microstructure which is otherwise very difficult, if not impossible, to approximate numerically.

In the following examples, $n=m=2, D=(-1,1)^{2}$ and a family of regular triangulations $\mathfrak{T}_{h}(D)$ for $h_{N}=2 \sqrt{2} / N$ with $N \geq 2$ is introduced by the lines

$$
\begin{cases}x=-1+\frac{2}{N} i, & 0 \leq i \leq N \\ y=-1+\frac{2}{N} j, & 0 \leq j \leq N \\ y=x-\frac{2}{N} k, & -N+1 \leq k \leq N-1\end{cases}
$$

For numerical solutions $\left(u_{h}, L_{h}\right) \in \mathbb{U}_{h} \times T_{h}(D)$, we denote

$$
\bar{F}_{h}=F\left(u_{h}, L_{h} ; D\right)
$$

which should converge to $Q f(A)$ (see (2.15), (2.27) and corollary 2.2), and denote

$$
\begin{gathered}
I_{h}(D)=\left(\int_{L_{h}(D)}\left|u_{h}\left(L_{h}^{-1}(x)\right)\right|^{2} d x\right)^{1 / 2}, \\
I_{h}(\partial D)=\left(\int_{\partial L_{h}(D)}\left|u_{h}\left(L_{h}^{-1}(x)\right)\right|^{2} d x\right)^{1 / 2},
\end{gathered}
$$

which are expected to be of the order of $h$ when the mesh scale oscillations are obtained.

Example 1. Let $\mathbf{a}=\left(-\frac{5}{8}, \frac{5}{6}\right)^{T}$ and $\mathbf{n}=\left(-\frac{4}{5}, \frac{3}{5}\right)^{T}$. Let

$$
B=\mathbf{a} \otimes \mathbf{n}=\left(\begin{array}{rr}
\frac{1}{2} & -\frac{3}{8} \\
-\frac{2}{3} & \frac{1}{2}
\end{array}\right) .
$$


Let $f: R^{2 \times 2} \rightarrow R$ be defined by

$$
f(\xi)=\langle\xi-B, \xi-B\rangle \cdot\langle\xi+B, \xi+B\rangle,
$$

where $\langle a, b\rangle=\operatorname{tr}\left(\xi^{T} \eta\right)$ is the inner product of $R^{2 \times 2}$. The linear boundary conditions $A(\lambda) x$ with

$$
A(\lambda)=\lambda B+(1-\lambda)(-B)=(2 \lambda-1) B, \quad 0<\lambda<1,
$$

are considered.

It is obvious that $B$ and $-B$, which are in rank one connection (see (3.6)), are the two potential wells of $F(u ; \Omega)$, and it is easily seen $[5,26]$ that

$$
Q F(A(\lambda))=0
$$

and the Young measure of a minimizing sequence of $F(\cdot ; \Omega)$ in $\mathbb{U}(A(\lambda) ; \Omega)$ is homogeneous and is given by

$$
\mu^{\lambda}(\xi)=\lambda \delta_{B}(\xi)+(1-\lambda) \delta_{-B}(\xi)
$$

where $\delta_{E}$ is the Dirac measure centered at $E$. Thus, the numerical solutions $\left(u_{h}^{\lambda}, L_{h}^{\lambda}\right) \in \mathbb{U}_{h}(D) \times T_{h}(D)$ to $(\mathrm{DPI})$ (or $\left.(\mathrm{DP})\right)$ are expected to satisfy $[5,26]$ (see also section 2)

$$
\begin{gathered}
A(\lambda)+\nabla u_{h}^{\lambda}\left(\nabla L_{h}^{\lambda}\right)^{-1} \rightarrow \mu^{\lambda}, \quad \text { in the sense of measure, } \\
F\left(u_{h}^{\lambda}, L_{h}^{\lambda} ; D\right) \rightarrow 0 .
\end{gathered}
$$

To reflect such a weak convergence of the numerical solutions, we use the following notations:

$$
\begin{gathered}
e_{\infty}\left(u_{h}^{\lambda}, L_{h}^{\lambda} ; K\right)=\min \left\{\left|A(\lambda)+\nabla u_{h}^{\lambda}\left(\nabla L_{h}^{\lambda}\right)^{-1} \pm B\right|\right\}, \quad \forall K \in \mathfrak{T}_{h}(D), \\
E_{2, h}^{\lambda}=\left(\sum_{K \subset C(k)}\left(e_{\infty}\left(u_{h}^{\lambda}, L_{h}^{\lambda} ; K\right)\right)^{2} \operatorname{meas}\left(L_{h}^{\lambda}(K)\right)\right)^{1 / 2}, \\
L_{h}^{ \pm}(D, \lambda)=\left\{x \in D:\left|A(\lambda)+\nabla u_{h}^{\lambda}(x)\left(\nabla L_{h}^{\lambda}(x)\right)^{-1} \mp B\right|=e_{\infty}\left(u_{h}^{\lambda}, L_{h}^{\lambda} ; K\right)\right\}, \\
e_{\lambda, h}=\max \left\{\left|\frac{\operatorname{meas}\left(L_{h}^{+}(D, \lambda)\right)}{\lambda}-1\right|,\left|\frac{\operatorname{meas}\left(L_{h}^{-}(D, \lambda)\right)}{1-\lambda}-1\right|\right\} .
\end{gathered}
$$

Numerical results with various $\lambda$ and $h=\sqrt{2} / 8(N=16)$ obtained by solving (DPI) with the penalty term (3.1), using the conjugate gradient method combined with the incremental crystallization and continuation techniques, are shown in table 1. The numerical results for a fixed $\lambda=0.6$ and $N=8,16,32$ are shown in table 2. In the computation, we set $\alpha h^{-r}=2.5, \beta h^{-q}=0.12$ and $\lambda_{0}=0.5$ initially, and when the norm of the gradient of the energy drops to $10^{-5}$ we set $\lambda$ to its final value and allow $\alpha h^{-r}$ and $\beta h^{-q}$ to decrease gradually to the order of 
$10^{-8}$ and $10^{-4}$ respectively as long as the increase of the corresponding integrals $I_{h}(D)$ and $I_{h}(\partial D)$ do not exceed 3 times of their original values.

\begin{tabular}{|c|c|c|c|c|c|}
\hline$\lambda$ & $\bar{F}_{h}$ & $E_{2, h}^{\lambda}$ & $e_{\lambda, h}$ & $I_{h}(D)$ & $I_{h}(\partial D)$ \\
\hline 0.5 & $1.30 \times 10^{-11}$ & $4.90 \times 10^{-6}$ & $1.60 \times 10^{-6}$ & $3.75 \times 10^{-2}$ & $1.28 \times 10^{-3}$ \\
\hline 0.6 & $7.47 \times 10^{-11}$ & $1.17 \times 10^{-5}$ & $1.02 \times 10^{-4}$ & $3.61 \times 10^{-2}$ & $3.99 \times 10^{-3}$ \\
\hline 0.7 & $1.17 \times 10^{-11}$ & $4.65 \times 10^{-6}$ & $267 \times 10^{-3}$ & $3.18 \times 10^{-2}$ & $8.66 \times 10^{-3}$ \\
\hline 0.8 & $4.39 \times 10^{-9}$ & $8.46 \times 10^{-5}$ & $7.43 \times 10^{-3}$ & $2.46 \times 10^{-2}$ & $1.39 \times 10^{-2}$ \\
\hline 0.9 & $3.94 \times 10^{-7}$ & $5.43 \times 10^{-4}$ & $1.79 \times 10^{-3}$ & $1.81 \times 10^{-2}$ & $3.47 \times 10^{-2}$ \\
\hline
\end{tabular}

TABLE 1. Numerical results for $N=16$ with $\mathrm{DPI}+\mathrm{p}+\mathrm{c}$.

\begin{tabular}{|c|c|c|c|c|c|}
\hline $\mathrm{N}$ & $\bar{F}_{h}$ & $E_{2, h}^{\lambda}$ & $e_{\lambda, h}$ & $I_{h}(D)$ & $I_{h}(\partial D)$ \\
\hline 8 & $1.21 \times 10^{-12}$ & $1.49 \times 10^{-6}$ & $2.58 \times 10^{-3}$ & $7.22 \times 10^{-2}$ & $1.17 \times 10^{-3}$ \\
\hline 16 & $7.47 \times 10^{-11}$ & $1.17 \times 10^{-5}$ & $1.02 \times 10^{-4}$ & $3.61 \times 10^{-2}$ & $3.99 \times 10^{-3}$ \\
\hline 32 & $1.52 \times 10^{-12}$ & $1.68 \times 10^{-6}$ & $3.70 \times 10^{-5}$ & $1.80 \times 10^{-2}$ & $1.80 \times 10^{-3}$ \\
\hline
\end{tabular}

TABLE 2. Numerical results for $\lambda=0.6$ with DPI $+\mathrm{p}+\mathrm{c}$.

\begin{tabular}{|c|c|c|c|c|c|}
\hline Method & $\bar{F}_{h}$ & $E_{2, h}^{\lambda}$ & $e_{\lambda, h}$ & $I_{h}(D)$ & $I_{h}(\partial D)$ \\
\hline DPI+p+c & $1.30 \times 10^{-11}$ & $4.90 \times 10^{-6}$ & $1.60 \times 10^{-6}$ & $3.75 \times 10^{-2}$ & $1.28 \times 10^{-3}$ \\
\hline DPI+p-c & $6.42 \times 10^{-4}$ & $3.46 \times 10^{-2}$ & $8.86 \times 10^{-3}$ & $6.50 \times 10^{-2}$ & $1.79 \times 10^{-1}$ \\
\hline DPI-p+c & $2.92 \times 10^{-12}$ & $2.32 \times 10^{-6}$ & $9.00 \times 10^{-4}$ & $3.62 \times 10^{-2}$ & $4.62 \times 10^{-3}$ \\
\hline DPI-p-c & $3.87 \times 10^{-4}$ & $2.67 \times 10^{-2}$ & $6.07 \times 10^{-3}$ & $6.84 \times 10^{-2}$ & $1.76 \times 10^{-1}$ \\
\hline DP+p+c & $8.18 \times 10^{-2}$ & $4.84 \times 10^{-1}$ & $1.05 \times 10^{-2}$ & $4.98 \times 10^{-2}$ & null \\
\hline DP-p+c & $1.26 \times 10^{-1}$ & $7.06 \times 10^{-1}$ & $4.86 \times 10^{-2}$ & $5.25 \times 10^{-2}$ & null \\
\hline DP+p-c & $8.18 \times 10^{-2}$ & $4.83 \times 10^{-1}$ & $1.05 \times 10^{-2}$ & $5.01 \times 10^{-2}$ & null \\
\hline DP-p-c & $1.06 \times 10^{-1}$ & $5.66 \times 10^{-1}$ & $1.06 \times 10^{-2}$ & $6.85 \times 10^{-2}$ & null \\
\hline
\end{tabular}

TABLE 3. Numerical results for $\lambda=0.6$ with various methods.

In table 3, we compare the numerical results obtained by solving (DPI) (or (DP)) with (or without) the penalty term (3.1) (referred to as \pm p respectively), using (or not using) the continuation technique (referred to as \pm c respectively), for $\lambda=0.6$ and $N=16$. 


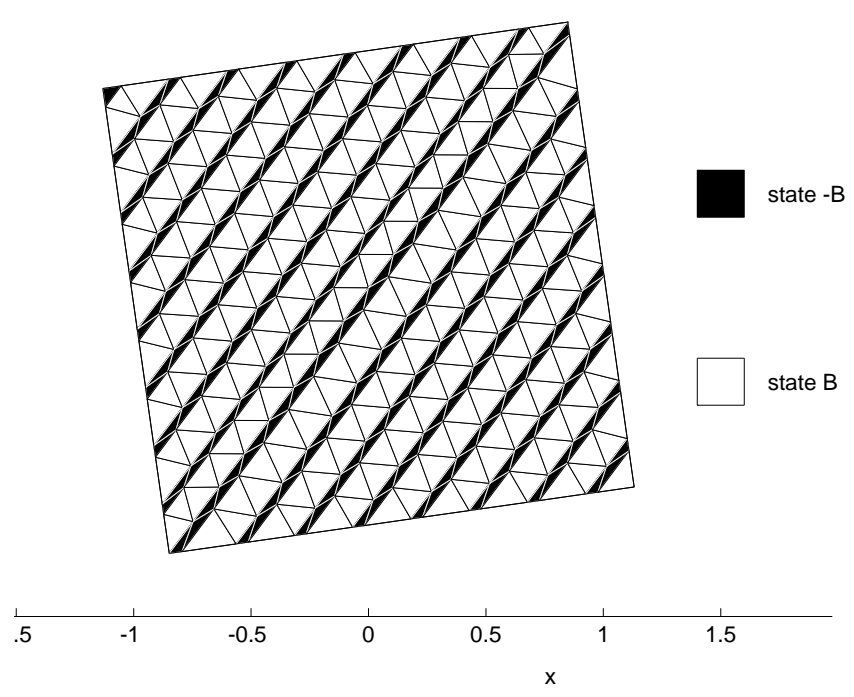

Figure 1. Numerical microstructure for $\lambda=0.8$ by DPI $+\mathrm{p}+\mathrm{c}$.
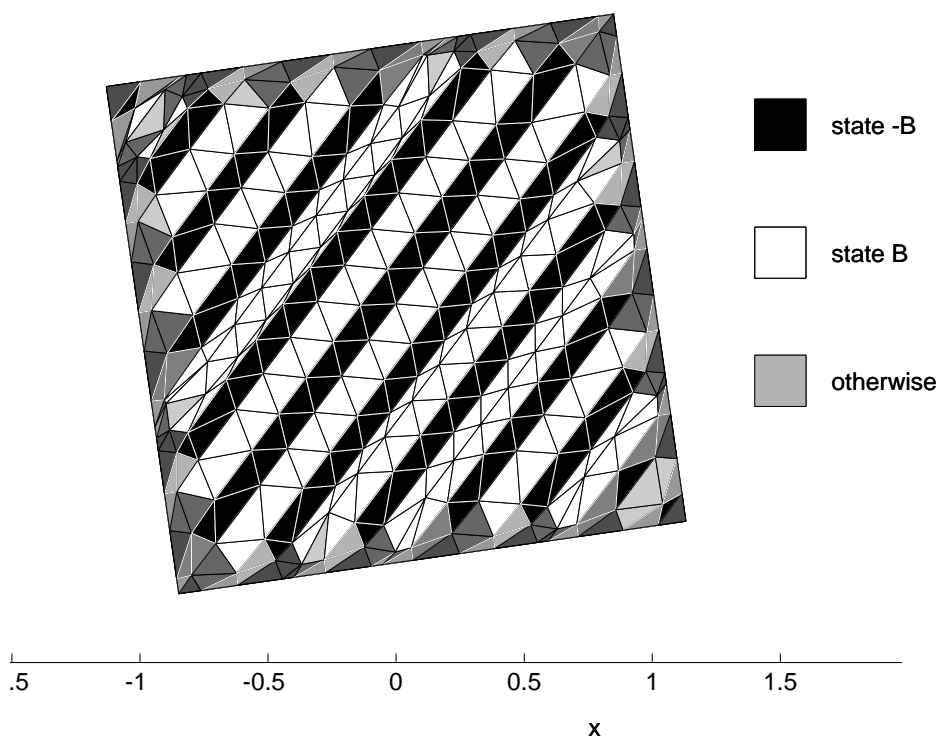

Figure 2. Numerical microstructure for $\lambda=0.6$ by $\mathrm{DP}-\mathrm{p}-\mathrm{c}$.

A numerical microstructure for $\lambda=0.8$ and $N=16$ obtained by (DPI+p+c) is shown in figure 1 and a numerical microstructure for $\lambda=0.6$ and $N=16$ obtained by (DP-p-c) is shown in figure 2. In both cases we can see clearly the advantage of the mesh transformation method. From numerical results shown in the tables and the figures, it is obvious that (DPI) works generally better than 
(DP), and the continuation technique also shows its power, especially in the case of (DPI) where the boundary effect is reduced to a minimum, and finally the penalty term (3.1) reduces the error $e_{\lambda, h}$ which means a better approximation to the Young measure $\mu^{\lambda}$.

Example 2. Consider an optimal design problem [26, 34, 35] where the integrand is given by

$$
f(\xi)= \begin{cases}1+|\xi|^{2}, & \text { if } \xi \neq 0, \\ 0, & \text { if } \xi=0 .\end{cases}
$$

It is known $[26,34,35]$ that in the case $n=m=2$

$$
Q f(\xi)= \begin{cases}1+|\xi|^{2}, & \text { if } \rho(\xi) \geq 1 \\ 2 \rho(\xi)-2|\operatorname{det} \xi|, & \text { if } \rho(\xi) \leq 1\end{cases}
$$

where

$$
\rho(\xi)=\left(|\xi|^{2}+2|\operatorname{det} \xi|\right)^{1 / 2}
$$

Let

$$
B=\left(\begin{array}{rr}
1 & -\frac{1}{2} \\
-\frac{1}{2} & \frac{1}{2}
\end{array}\right)
$$

We consider the affine boundary conditions associate with matrices

$$
A(\lambda)=\lambda B=\left(\begin{array}{rr}
\lambda & -\frac{\lambda}{2} \\
-\frac{\lambda}{2} & \frac{\lambda}{2}
\end{array}\right) .
$$

By (3.14),

$$
Q f(A(\lambda))= \begin{cases}1+\frac{7}{4} \lambda^{2}, & \text { if }|\lambda| \geq \frac{2}{3} \\ \frac{1}{2}|\lambda|(6-|\lambda|), & \text { if }|\lambda| \leq \frac{2}{3}\end{cases}
$$

Since there is a removable discontinuity point at the very heart, the unique potential well of the integrand, $f$ given by (3.13) is not a nice function for numerical approximations. So, we consider of an application of the continuation method, which is essential to the numerical approximations. Instead of dealing with $f$, we introduce a sequence of integrands $f_{k}: R^{2 \times 2} \rightarrow R, k=1,2, \ldots$,

$$
f_{k}(\xi)= \begin{cases}1+|\xi|^{2}, & \text { if }|\xi| \geq \frac{1}{k} \\ \left(1+k^{2}\right)|\xi|^{2}, & \text { if }|\xi| \leq \frac{1}{k}\end{cases}
$$


It is easily seen that

$$
Q f-2 \sqrt{2} \frac{1}{k^{2}} \leq f_{k} \leq f_{k+1} \leq f, \quad \forall k .
$$

Hence, for all open set $\Omega \in R^{2}$ and $A \in R^{2 \times 2}$, we have [26]

$$
\lim _{k \rightarrow \infty} \inf _{u \in \mathbb{U}(A ; \Omega)} F_{k}(u ; \Omega)=\inf _{u \in \mathbb{U}(A ; \Omega)} F(u ; \Omega),
$$

where $F_{k}(u ; \Omega)=\int_{\Omega} f_{k}(\nabla u(x)) d x$.

Let $u_{k} \in \mathbb{U}(A ; \Omega)$ satisfy

$$
F_{k}\left(u_{k} ; \Omega\right) \leq \inf _{u \in \mathbb{U}(A ; \Omega)} F_{k}(u ; \Omega)+\varepsilon_{k},
$$

with $\lim _{k \rightarrow \infty} \varepsilon_{k}=0$. Then, it follows from (3.20)-(3.22) that

$$
\limsup _{k \rightarrow \infty} F_{i}\left(u_{k} ; \Omega\right) \leq \inf _{u \in \mathbb{U}(A ; \Omega)} F(u ; \Omega) .
$$

Since, by (3.23) and (h1), $\left\{u_{k}\right\}_{k=1}^{\infty}$ are uniformly bounded in $W^{1, p}\left(\Omega ; R^{2}\right)$ and since a affine boundary condition is considered, without loss of generality, we may assume that there exists a homogeneous Young measure $\mu$ on $R^{2 \times 2}$, which is a probability measure [5], such that

$$
\lim _{k \rightarrow \infty} F_{i}\left(u_{k} ; \Omega\right)=\int_{\Omega}\left\langle f_{i}(\cdot), \mu\right\rangle d x=\operatorname{meas}(\Omega)\left\langle f_{i}(\cdot), \mu\right\rangle, \quad \forall i
$$

On the other hand, for a probability measure $\mu$ on $R^{2 \times 2}$, it follows from

$$
0 \leq\left\langle f(\cdot)-f_{i}(\cdot), \mu\right\rangle \leq \mu\left(\left\{\xi \in R^{2 \times 2}: 0<|\xi|<i^{-1}\right\}\right),
$$

and

$$
\lim _{i \rightarrow \infty} \mu\left(\left\{\xi: 0<|\xi|<i^{-1}\right\}\right)=\mu\left(\bigcap_{i=1}^{\infty}\left\{\xi: 0<|\xi|<i^{-1}\right\}\right)=\mu(\emptyset)=0 .
$$

Combining (3.23)-(3.26), we obtain

$$
\int_{\Omega}\langle f(\cdot), \mu\rangle d x=\inf _{u \in \mathbb{U}(A ; \Omega)} F(u ; \Omega) .
$$

The above analysis indicates that while a sequence $\left\{u_{k}\right\}_{k=1}^{\infty}$, which satisfies (3.22) and has a homogeneous Young measure representation $\mu$, is generally not a minimizing sequence of $F(\cdot ; \Omega)$ in $\mathbb{U}(A ; \Omega)$, a modification can be made to $\left\{u_{k}\right\}_{k=1}^{\infty}$ to produce such a minimizing sequence which has the Young measure representation $\mu$. 
In our numerical experiments the method $(\mathrm{DPI}+\mathrm{p})$, i.e. (DPI) with the penalty term (3.1), is applied. To solve the discrete problem for large $k$, the continuation method is again applied. We either take $\lambda$ as a parameter or consider the parameterized integrands

$$
f_{k, l}(\xi)= \begin{cases}1+|\xi|^{2}, & \text { if }|\xi| \geq \frac{l}{k} \\ \left(1+l^{-2} k^{2}\right)|\xi|^{2}, & \text { if }|\xi| \leq \frac{l}{k}\end{cases}
$$

The parameters $\lambda$ or $l$ in (3.28) will be varied incrementally from their initial values, say $\lambda_{0}=0.025$ and $l_{0}=\frac{k}{1+\|A(\lambda)\|}$, to the final value of $\lambda$ and 1 respectively, and in each step a convergent criterion, say the norm of the energy gradient less than $10^{-4}$, is to be satisfied. Both of the processes, encourage the deformation gradients to fall into the energy well, which is very small for large $k$, and thus greatly improve the performance of the algorithm. It should be brought into attention here however that the value $k$ is limited by the mesh size $h$, in other words, to solve the problem with large $k$ the mesh size $h$ must be sufficiently small. In showing the numerical results, we denote the relative error of $\bar{F}_{h}$ by $E_{r}\left(\bar{F}_{h}\right)$ and define

$$
\begin{gathered}
C(k)=\left\{\xi \in R^{2 \times 2}:|\xi| \leq \frac{1}{k}\right\}, \\
e_{\infty}\left(u_{h}^{\lambda}, L_{h}^{\lambda} ; K\right)=\mid A(\lambda)+\nabla u_{h}^{\lambda}\left(\nabla L_{h}^{\lambda}\right)^{-1} \|_{K}, \text { for } K \subset C(k), \\
E_{\infty, h}^{\lambda}(C(k))=\max _{K \subset C(k)} e_{\infty}\left(u_{h}^{\lambda}, L_{h}^{\lambda} ; K\right), \\
E_{2, h}^{\lambda}(C(k))=\left(\sum_{K \subset C(k)}\left(e_{\infty}\left(u_{h}^{\lambda}, L_{h}^{\lambda} ; K\right)\right)^{2} \operatorname{meas}\left(L_{h}^{\lambda}(K)\right)\right)^{1 / 2} .
\end{gathered}
$$

In table 4, numerical results for $k=30, N=16$ and various $\lambda$ are shown. Table 5 shows the numerical results for $\lambda=0.3, k=30$ and $N=8,16,32,64$, and it is clearly shown that $I_{h}(D)$ and $I_{h}(\partial D)$ decrease as $N$ increases (or equivalently as $h$ decreases), this means the weakly convergence of the finite element solutions. Table 6 shows the numerical results for $N=16, \lambda=0.3$ and $k=15,30,60$. In table 6 , we see that $E_{r}\left(\bar{F}_{h}\right)$ decreases as $k$ increases, this indicates the finite element solutions satisfy (3.21), we see also the gradients in the wells $C(k)$ (see $E_{\infty, h}^{\lambda}(C(k))$ and $\left.E_{2, h}^{\lambda}(C(k))\right)$ converge sharply to 0 as $k$ increases, this implies the convergence of the Young measure.

Numerical microstructures for $\lambda=0.3$ obtained by the algorithm with different initial data and the combinations of the rotation transformation method, the incremental crystallization method and the continuation method are shown in 


\begin{tabular}{|c|c|c|c|c|c|}
\hline$\lambda$ & $E_{r}\left(\bar{F}_{h}\right)$ & $E_{\infty, h}^{\lambda}(C(k))$ & $E_{2, h}^{\lambda}(C(k))$ & $I_{h}(D)$ & $I_{h}(\partial D)$ \\
\hline 0.05 & $7.62 \times 10^{-2}$ & $9.93 \times 10^{-3}$ & $6.10 \times 10^{-5}$ & $1.79 \times 10^{-2}$ & $4.21 \times 10^{-2}$ \\
\hline 0.15 & $3.08 \times 10^{-3}$ & $3.04 \times 10^{-2}$ & $1.02 \times 10^{-4}$ & $3.10 \times 10^{-2}$ & $7.15 \times 10^{-2}$ \\
\hline 0.25 & $5.93 \times 10^{-2}$ & $2.65 \times 10^{-2}$ & $1.09 \times 10^{-4}$ & $3.72 \times 10^{-2}$ & $7.00 \times 10^{-2}$ \\
\hline 0.35 & $6.01 \times 10^{-2}$ & $2.67 \times 10^{-2}$ & $1.66 \times 10^{-4}$ & $4.48 \times 10^{-2}$ & $7.36 \times 10^{-2}$ \\
\hline 0.45 & $2.51 \times 10^{-2}$ & $2.62 \times 10^{-2}$ & $1.44 \times 10^{-4}$ & $4.57 \times 10^{-2}$ & $1.16 \times 10^{-1}$ \\
\hline 0.55 & $1.11 \times 10^{-2}$ & $3.32 \times 10^{-2}$ & $1.40 \times 10^{-4}$ & $4.31 \times 10^{-2}$ & $1.22 \times 10^{-1}$ \\
\hline
\end{tabular}

TABLE 4. Numerical results for $N=16$ and $k=30$.

\begin{tabular}{|c|c|c|c|c|c|}
\hline $\mathrm{N}$ & $E_{r}\left(\bar{F}_{h}\right)$ & $E_{\infty, h}^{\lambda}(C(k))$ & $E_{2, h}^{\lambda}(C(k))$ & $I_{h}(D)$ & $I_{h}(\partial D)$ \\
\hline 8 & $2.92 \times 10^{-2}$ & $1.89 \times 10^{-2}$ & $2.77 \times 10^{-5}$ & $6.33 \times 10^{-2}$ & $9.75 \times 10^{-2}$ \\
\hline 16 & $4.13 \times 10^{-2}$ & $2.17 \times 10^{-2}$ & $7.93 \times 10^{-5}$ & $3.85 \times 10^{-2}$ & $6.42 \times 10^{-2}$ \\
\hline 32 & $4.58 \times 10^{-2}$ & $2.53 \times 10^{-2}$ & $6.17 \times 10^{-5}$ & $2.42 \times 10^{-2}$ & $4.51 \times 10^{-2}$ \\
\hline 64 & $6.37 \times 10^{-2}$ & $2.72 \times 10^{-2}$ & $1.44 \times 10^{-4}$ & $1.39 \times 10^{-2}$ & $3.23 \times 10^{-2}$ \\
\hline
\end{tabular}

TABLE 5. Numerical results for $\lambda=0.3$ and $k=30$.

\begin{tabular}{|c|c|c|c|c|c|}
\hline $\mathrm{k}$ & $E_{r}\left(\bar{F}_{h}\right)$ & $E_{\infty, h}^{\lambda}(C(k))$ & $E_{2, h}^{\lambda}(C(k))$ & $I_{h}(D)$ & $I_{h}(\partial D)$ \\
\hline 15 & $8.37 \times 10^{-2}$ & $5.67 \times 10^{-2}$ & $1.09 \times 10^{-3}$ & $3.31 \times 10^{-2}$ & $4.87 \times 10^{-2}$ \\
\hline 30 & $6.78 \times 10^{-2}$ & $2.73 \times 10^{-2}$ & $1.78 \times 10^{-4}$ & $4.33 \times 10^{-2}$ & $7.23 \times 10^{-2}$ \\
\hline 60 & $2.76 \times 10^{-2}$ & $8.75 \times 10^{-3}$ & $8.49 \times 10^{-6}$ & $4.69 \times 10^{-2}$ & $8.33 \times 10^{-2}$ \\
\hline
\end{tabular}

TABLE 6. Numerical results for $\lambda=0.3$ and $N=16$.

figure 3 - figure 8 . It can be clearly seen, as is expected since the Young measure is wildly nonunique in this case $[34,35]$, that these numerical microstructures are not simple laminates and are quite different from each other. 
y
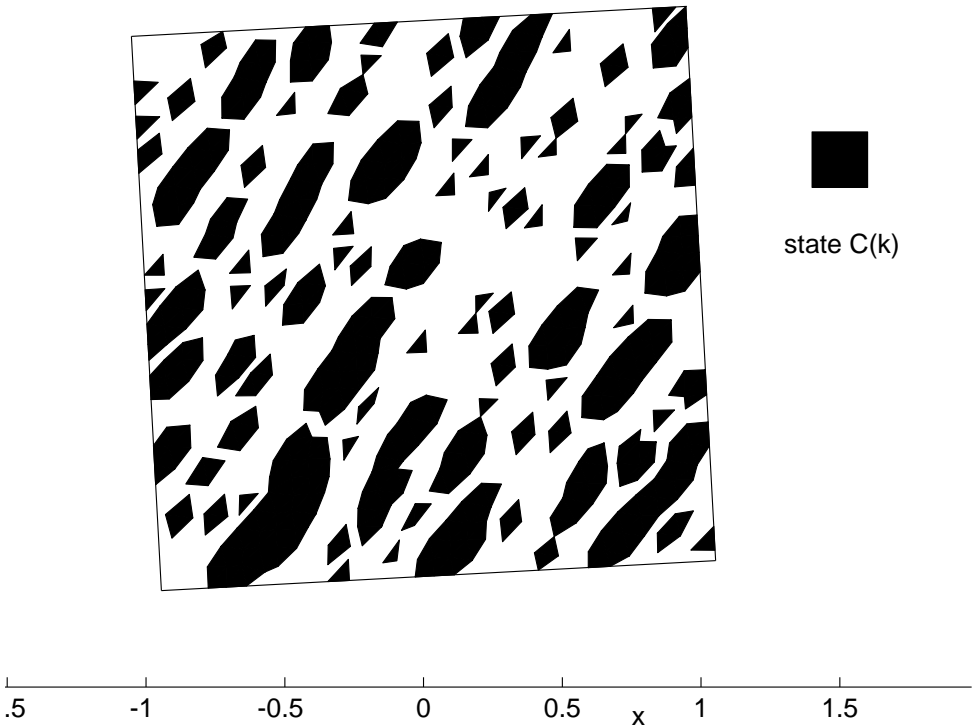

FiguRE 3. A numerical microstructure for $\lambda=0.3, k=30$.

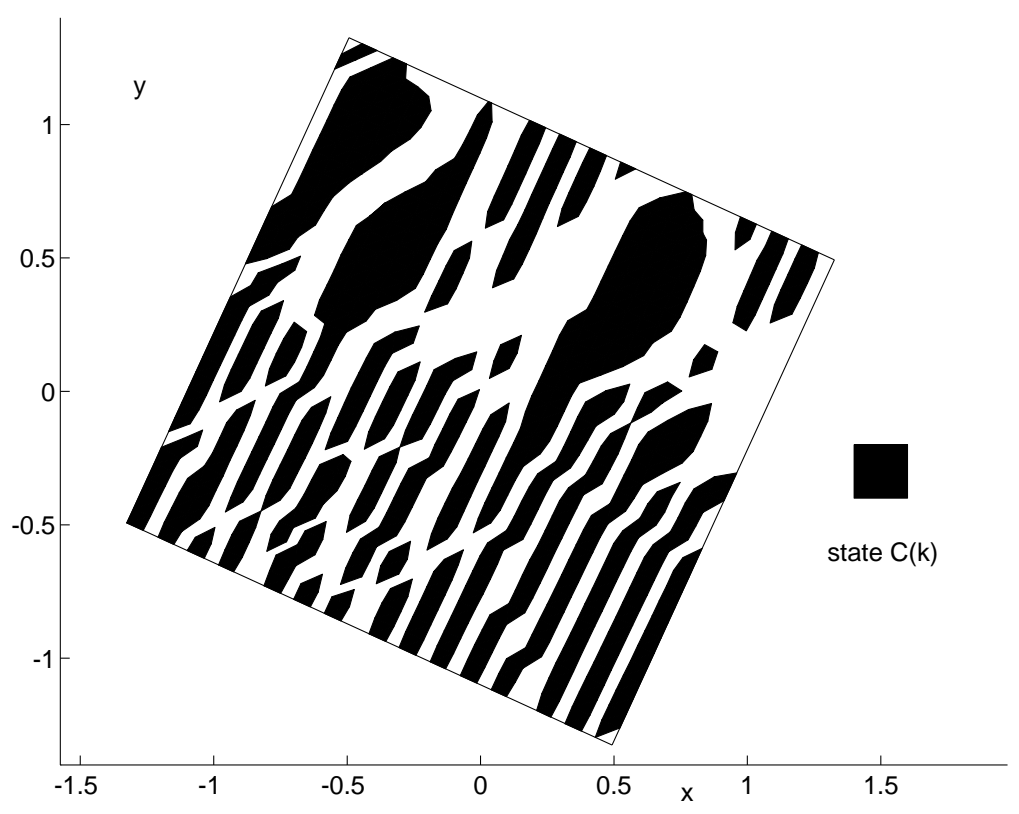

Figure 4. A numerical microstructure for $\lambda=0.3, k=30$. 


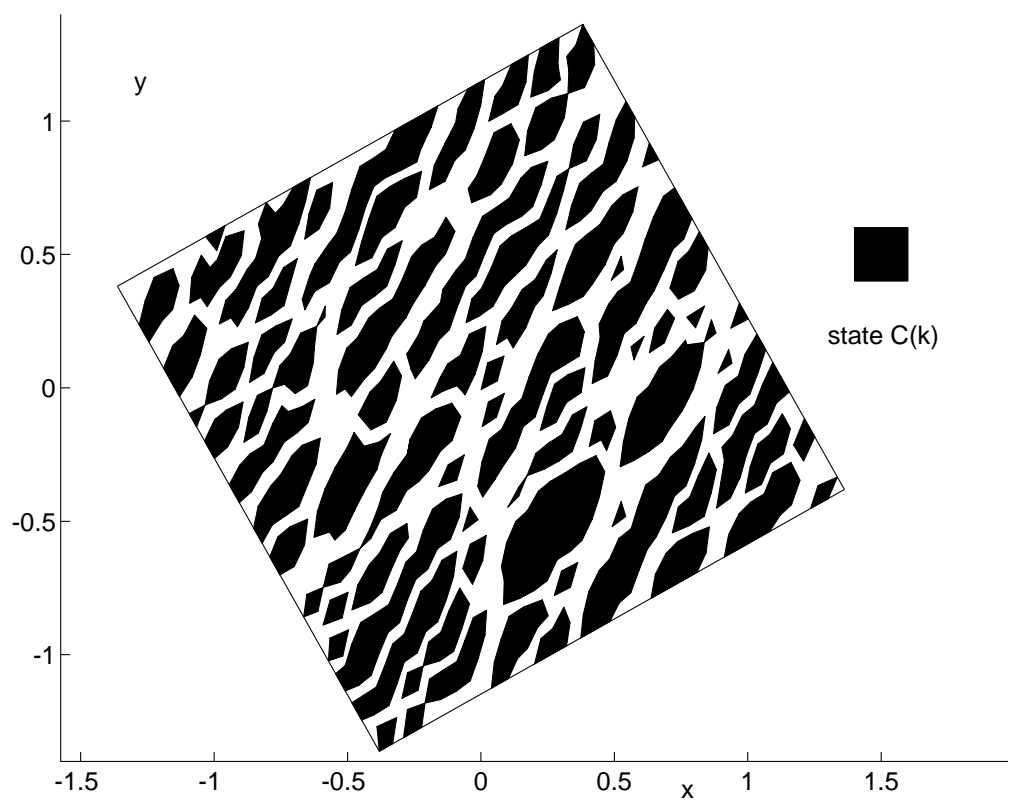

Figure 5. A numerical microstructure for $\lambda=0.3, k=30$.

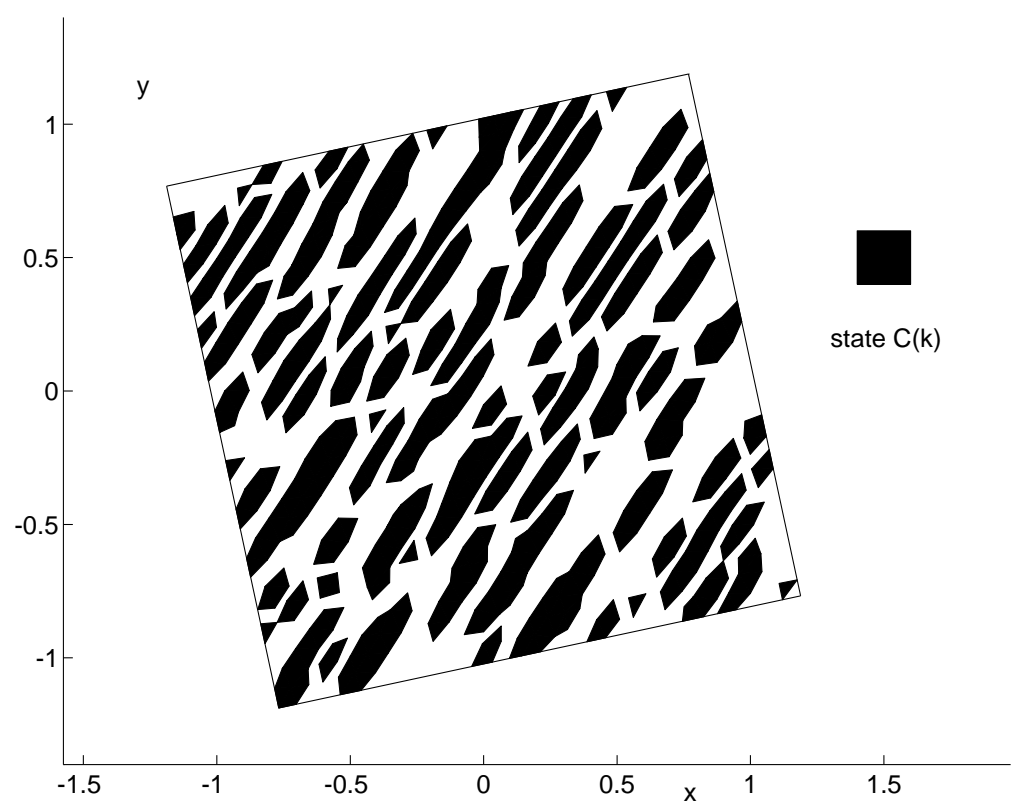

Figure 6. A numerical microstructure for $\lambda=0.3, k=30$.

While the numerical microstructures shown in figure 3 - figure 7 are obtained by $N=32$ and $k=30$, the numerical microstructure in figure 8 is obtained by $N=64$ and $k=60$. We see that the $\operatorname{mesh}_{21}$ size oscillations are obtained. 


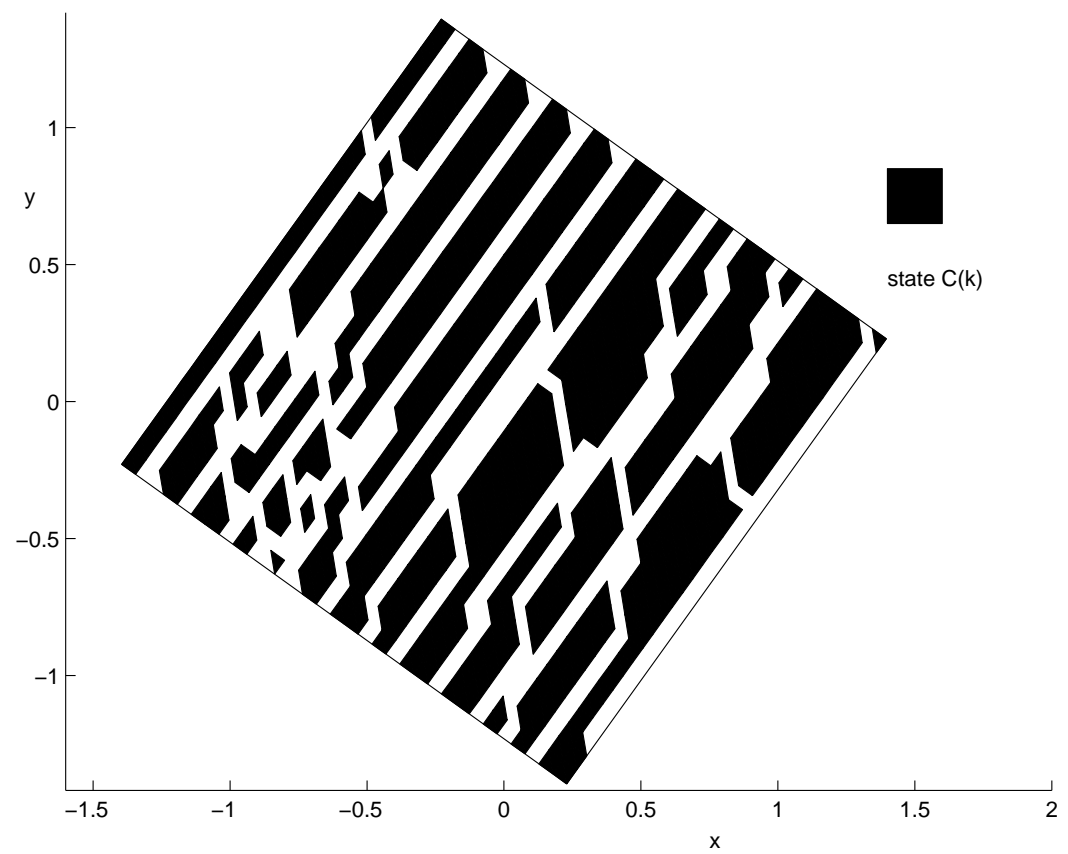

FiguRE 7. A numerical microstructure for $\lambda=0.3, k=30$.

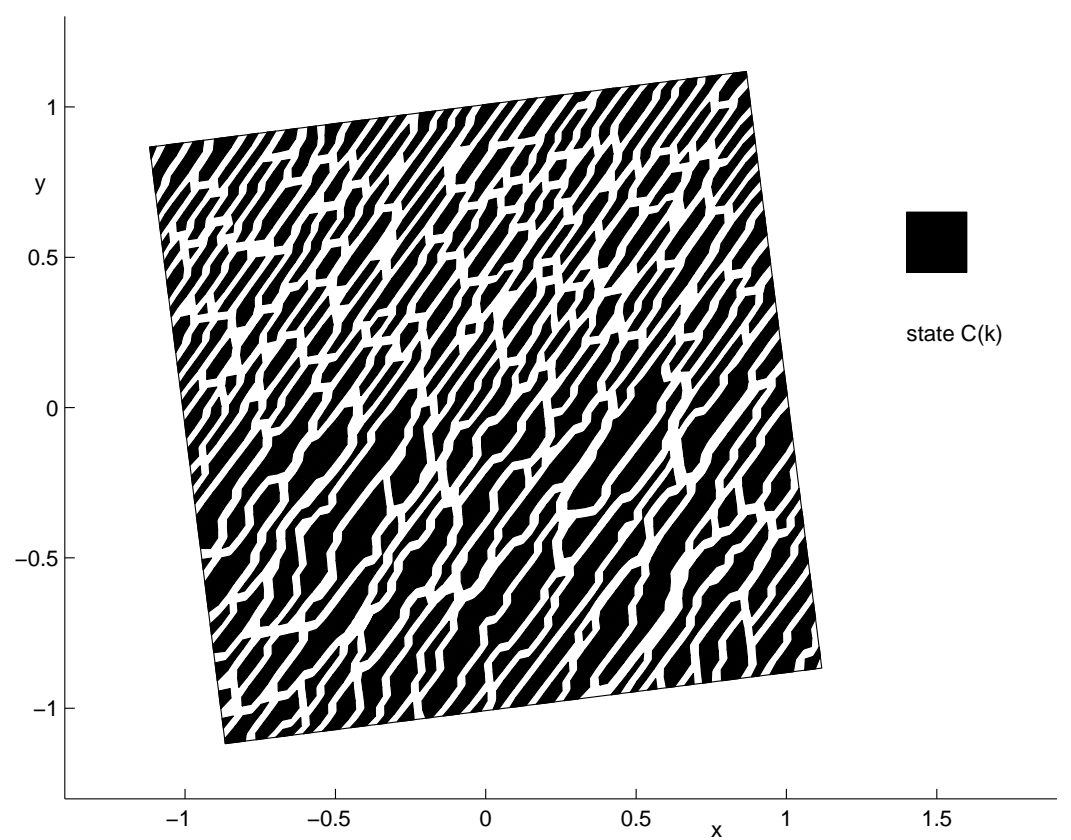

Figure 8. A numerical microstructure for $\lambda=0.3, k=60$.

\section{REFERENCES}

[1] J. M. Ball and R. D. James, Fine phase mixtures as minimizers of energy. Arch. Rat. Mech. Anal., 100 (1987)1, 13-52. 
[2] Y. Hou, I. Müler and S. Seelecke, Quasiplasticity and pseudoelasticity in shape memory alloys, in Phase Transitions and Hysteresis, ed. A. Vistini, Lecture Notes in Mathematics, No. 1584, Springer-Verlag, Berlin, pp.87-146.

[3] Z.-P. Li, Existence of minimizers and microstructures in nonlinear elasticity. Nonlinear Anal., 27 (1996), 297-308.

[4] Z.-P. Li, Laminated microstructure in a variational problem with a non-rank-one connected double well potential, J. Math. Anal. Appl., 217(1998), 490-500.

[5] J.M. Ball, A version of the fundamental theorem for Young measures. In Partial Differential Equations and Continuum models of Phase Transitions, Lecture Notes in Physics, No. 344, M. Rascle, D. Serre and M. Slemrod, eds., Springer-Verlag, New York (1989), pp. 207-215.

[6] C. Collins and M Luskin, The computation of the austenitic-martensitic phase transition. In Partial Differential Equations and Continuum Models of Phase Transitions Lecture Nodes in Physics, Vol. 344, M. Rascle, D. Serre and M. Slemrod, eds, SpringerVerlag, New York, (1989), pp. 34-50.

[7] C. Collins, D. Kinderlehrer and M Luskin, Numerical approximation of the solution of a variational problem with a double well potential. SIAM J. Numer. Anal., 28(1991), 321-332.

[8] M. Chipot, Numerical analysis of oscillations in nonconvex problems. Numer. Math., 59(1991), 747-767.

[9] C. Collins, Computation of Twinning. In Microstructure and Phase Transitions, IMA Volumes in Mathematics and Its Applications, Vol.54, J. Ericksen, R. James, D. Kinderlehrer and M. Luskin, eds, Springer-Verlag, New York, (1993), pp. 39-50.

[10] C. Collins, M. Luskin and J. Riordan, Computational results for a two-dimensional model of crystalline microstructure, in Microstructure and Phase Transitions, IMA Volumes in Mathematics and Its Applications, Vol.54, J. Ericksen, R. James, D. Kinderlehrer and M. Luskin, eds, Springer-Verlag, New York, (1993), pp. 51-56.

[11] M. Luskin and L. Ma, Numerical optimization of the micromagnetics energy, in Mathematics in Smart Materials, SPIE, (1993), pp. 19-29.

[12] P. Gremaud, Numerical optimization and quasiconvexity, Euro. J. Appl. Math. 6, (1995) 69-82.

[13] P. Pedregal, Numerical approximation of parameterized measures, Numer. Funct. Anal. Optimiz., 16(1995), 1049-1066.

[14] P. Pedregal, On the numerical analysis of non-convex variational problems, Numer. Math., 74(1996), 325-336.

[15] M. Luskin, On the computation of crystalline microstructure. Acta Numerica, 1996.

[16] Z.-P. Li, Simultaneous numerical approximation of microstructures and relaxed minimizers. Numer. Math., 78 (1997), 21-38.

[17] R. A. Nicolaides, N. Walkington and H. Wang, Numerical methods for a nonconvex optimization problem modeling martensitic microstructure. SIAM J. Sci. Comput., 18(1997), 1122-1141. 
[18] C. Carstensen and P. Plecháč, Numerical solution of the scalar double-well problem allowing microstructure. Math. Comput., 66(1997), 997-1026.

[19] C. R. Collins, Convergence of a reduced integration method for computing microstructures. SIAM J. Numer. Anal., 35(1998), 1271-1298.

[20] M. Kružík, Numerical approach to double well problems. SIAM J. Numer. Anal., 35(1998), 1833-1849.

[21] Z.-P. Li, Rotational transformation method and some numerical techniques for the computation of microstructures. Math. Models Meth. Appl. Sci., 8(1998), 985-1002.

[22] Z.-P. Li, Finite order rank-one convex envelopes and computation of microstructures with laminates in laminates. Research Report No.78, Institute of Mathematics and School of Mathematical Sciences, Peking University, 1998.

[23] Z.-P. Li, A periodic relaxation method for computing microstructures. Appl. Numer. Math., 32(2000), 291-303.

[24] Z.-P. Li, Computations of needle-like microstructures. Research Report No.71, Institute of Mathematics and School of Mathematical Sciences, Peking University, 1999.

[25] C. B. Morrey, Multiple Integrals in the Calculus of Variations. Springer-Verlag, New York, 1966.

[26] B. Dacorogna, Direct Methods in the Calculus of Variations, Applied Mathematical Sciences, 78, Springer-Verlag, Berlin, 1989.

[27] J. M. Ball and F. Murat $W^{1, p}$-quasiconvexity and variational problems for multiple integrals. J. Funct. Anal., 58(1984), 225-253.

[28] P.G. Ciarlet, The Finite Element Method for Elliptic Problems. North-Holland, Amsterdam, 1978.

[29] I. Diestel and J. J. Uhl, Jr., Vector Measures, American Mathematical Society, Mathematical Surveys No.15. Providence, 1977.

[30] R.A. Adams, Sobolev Spaces. Academic Press, New York, 1975.

[31] Z.-P. Li, An integral representation theorem on lower semicontinuous envelopes of integral functionals. Nonlinear Anal., 32(1998), 541-548.

[32] D. Kinderlehrer and P. Pedregal, Characterizations of Young measures generated by gradients. Arch. Rational Mech. Anal., 115(1991), 329-365.

[33] D. Kinderlehrer and P. Pedregal, Weak convergence of integrands and the Young measure representation. SIAM J. Math. Anal., 23(1992), 1-19.

[34] R. V. Kohn and G. Strang, Explicit relaxation of a variational problem in optimal design. Bull. A. M. S., 9(1983), 211-214.

[35] R. V. Kohn and G. Strang, Optimal design and relaxation of variational problems, I, II and III, Comm. Pure Appl. Math., 39(1986), 113-137, 139-182 and 353-377.

E-mail address: zpli@math.pku.edu.cn 